\title{
Numerical Solution of Nonlinear Singular Ordinary Differential Equations Arising in Biology Via Operational Matrix of Shifted Legendre Polynomials
}

\author{
K. Maleknejad ${ }^{*}$ E. Hashemizadeh \\ Department of Mathematics, Karaj Branch, Islamic Azad University, Karaj, Iran
}

\begin{abstract}
This paper proposed a numerical method for nonlinear singular ordinary differential equations, that arises in biology and some diseases. We solved these nonlinear problems by a new method based on shifted Legendre polynomials. Operational matrices of derivatives for this function are presented to reduce the nonlinear singular boundary value problems to a system of nonlinear algebraic equations. The method is computationally very simple and attractive, and applications are demonstrated through illustrative examples. The results obtained are compared by the known results.
\end{abstract}

Keywords Nonlinear Singular Boundary Value Problem, Legendre Polynomials, Operational Matrix of Derivative, Collocation Method, Biology

\section{Introduction}

The aim of this paper is to introduce a new method for the numerical solution of the following class of singular boundary value problems

$$
\begin{gathered}
y^{\prime \prime}(x)+\left(a+\frac{m}{x}\right) y^{\prime}=f(x, y), \quad 0 \leq x \leq 1, \\
\alpha_{1} y(0)+\beta_{1} y^{\prime}(0)=\gamma_{1}, \\
\alpha_{2} y(1)+\beta_{2} y^{\prime}(1)=\gamma_{2},
\end{gathered}
$$

which arising in biology and physiology problems. We assume that $f(x, y)$ is continuous, $\partial f / \partial y$ exists and is continuous and also $\partial f / \partial y \geq 0, \forall x 0 \leq x \leq 1$. The boundary value problem (1)-(3) with $m=0,1,2$ and $a=0$ arise in the study of various tumor growth problems, see ([1-6]), with linear $f(x, y)$ and with nonlinear $f(x, y)$ of the form

$$
f(x, y) \equiv f(y)=\frac{n y}{y+\mu}, \quad n>0, \mu>0,
$$

A mathematical model of tumor growth is a mathematical expression of the dependence of tumor size on time.

And when $m=2, a=0$ in the study of oxygen diffusion problem in a spherical cell with Michaelis-Menten Kinetics, see ([7-9]). A similar problem arise with $m=2$

* Corresponding author:

maleknejad@iust.ac.ir (K. Maleknejad)

Published online at http://journal.sapub.org/ajcam

Copyright (C) 2011 Scientific \& Academic Publishing. All Rights Reserved and $a=0$ in modelling of heat conduction in human head, see[10-13], with $f(x, y)$ of the form

$$
f(x, y) \equiv f(y)=-\delta e^{-\sigma y}, \sigma>0, \delta>0 .
$$

Existence-uniqueness results for such problems have been established by several researchers[14-16]. In recent years, finding numerical solutions of singular differential equations, particularly those arising in physiology, has been the focus of a number of authors, which you can see some of them in[17-20].

The purpose of this paper is to introduce a novel method based on operational matrices of derivatives of shifted Legandre polynomials that have been introduced recently in Saadatmandi and Dehghan work's[21] for the numerical solution of the class of singular second-order boundary value problems given in the (1-3) that arise in physiology. In this work by use of shifted Legendre polynomials as basis and operational matrices of derivatives of them we convert these kinds of equations to algebraic equations. The advantage of this method analogy to other existed method for these problems is its trusty and simply in implementation, we compared our results with some existed results to prove this claim.

This paper is organized as follows: Section 2 represents preliminaries, in this section we introduced shifted Legendre polynomials, and some properties of them, specially the operational matrices of derivatives, in Section 3 we implemented them on physiology problems. In Section 4, a number of applied models in physiology are discussed to show the efficiency and accuracy of the proposed method, the results obtained are compared by the known results. Finally, Section 5 includes a conclusion for the paper. 


\section{Definitions and Properties of Shifted Legendre Polynomials}

\subsection{Shifted Legendre Polynomials}

Consider the Legendre polynomials $L_{m}(z)$ on the interval $[-1,1]$

$$
L_{0}(z)=1, \quad L_{1}(z)=z,
$$

the set $\left\{L_{m}(z): m=0,1, \ldots\right\}$ in Hilbert space $L^{2}[-1,1]$ is a complete orthogonal set[22,23]. In order to use these polynomials on the interval $x \in[0,1]$ we define the so-called shifted Legendre polynomials by introducing the change of variable $z=2 x-1$. Let the shifted Legendre polynomials $L_{i}(2 x-1)$ be denoted by $P_{i}(x)$. Then $P_{i}(x)$ can be obtained as follows:

$$
P_{i+1}(x)=\frac{(2 i+1)(2 x-1)}{(i+1)} P_{i}(x)-\frac{i}{i+1} P_{i-1}(x), i=1,2, \ldots,
$$

where $P_{0}(x)=1$ and $P_{1}(x)=2 x-1$. The analytic form of the shifted Legendre polynomials $P_{i}(x)$ of degree $i$ given by

$$
P_{i}(x)=\sum_{k=0}^{i}(-1)^{i+k} \frac{(i+k) !}{(i-k) !} \frac{x^{k}}{(k !)^{2}} .
$$

Note that $P_{i}(0)=(-1)^{i}$ and $P_{i}(1)=1$. The orthogonality condition is

$$
\int_{0}^{1} P_{i}(x) P_{j}(x) d x= \begin{cases}\frac{1}{2 i+1}, & \text { for } i=j, \\ 0, & \text { for } i \neq j\end{cases}
$$

\subsection{Function Approximation}

Any function $y(x) \in L^{2}[0,1]$ can be expanded in terms of shifted Legendre polynomials as

$$
y(x)=\sum_{j=1}^{\infty} c_{j} P_{j}(x),
$$

where the coefficients $c_{j}$ are given by

$$
c_{j}=(2 j+1) \int_{0}^{1} y(x) P_{j}(x) d x, \quad, j=1,2, \ldots .
$$

In practice, only the first $(m+1)$-terms shifted Legendre polynomials are considered. Then we have

$$
y(x) \simeq \sum_{j=0}^{m} c_{i} P_{j}(x)=\mathbf{C}^{T} \mathbf{B}(x),
$$

where the shifted Legendre coefficient vector $\mathbf{C}$ and the shifted Legendre vector $\mathbf{B}$ are given by:

$$
\begin{aligned}
\mathbf{C} & =\left[c_{0}, c_{1}, \ldots, c_{m}\right]^{T}, \\
\mathbf{B}(x) & =\left[P_{0}(x), P_{1}(x), \ldots, P_{m}(x)\right]^{T} .
\end{aligned}
$$

\subsection{Operational Matrix of Derivative}

The derivative of the vector $\mathbf{B}(x)$ can be expressed by

$$
\frac{d \mathbf{B}(x)}{d x}=\mathbf{D}^{(1)} \mathbf{B}(x)
$$

where $\mathbf{D}^{(1)}$ is the $(m+1) \times(m+1)$ operational matrix of derivative given by]21]

$$
\mathbf{D}^{(1)}=\left(d_{i j}\right)=\left\{\begin{array}{cc}
2(2 j+1), & \text { forj }=i-k, \\
0, & \text { otherwise, }
\end{array}\right.
$$

as if $m$ is odd $k=1,3, \ldots, m$ and if $m$ is even $k=1,3, \ldots, m-1$. For example for even $m$ we have

$$
\mathbf{D}^{(1)}=2\left(\begin{array}{cccccccc}
0 & 0 & 0 & 0 & \ldots & 0 & 0 & 0 \\
1 & 0 & 0 & 0 & \ldots & 0 & 0 & 0 \\
0 & 3 & 0 & 0 & \ldots & 0 & 0 & 0 \\
1 & 0 & 5 & 0 & \ldots & 0 & 0 & 0 \\
\vdots & \vdots & \vdots & \vdots & \vdots & \vdots & \vdots & \vdots \\
1 & 0 & 5 & 0 & \ldots & 2 m-3 & 0 & 0 \\
0 & 3 & 0 & 7 & \ldots & 0 & 2 m-1 & 0
\end{array}\right) .
$$

By using Eq. (12), it is clear that

$$
\frac{d^{n} \mathbf{B}(x)}{d x^{n}}=\left(\mathbf{D}^{(1)}\right)^{n} \mathbf{B}(x),
$$

where $n \in \mathbb{N}$ and the superscript, in $\mathbf{D}^{(1)}$, denote matrix powers. Thus

$$
\mathbf{D}^{(n)}=\left(\mathbf{D}^{(1)}\right)^{n}, \quad n=1,2, \ldots
$$

\section{Implementation of Shifted Legendre Polynomials Method on Physiology Problems}

In this section we solve nonlinear singular boundary value problem of the form Eq.(1) with the mixed conditions (2) and (3) by using shifted Legendre polynomials.

From Eq. (9) we can approximate our unknown as

$$
y(x)=\mathbf{C}^{T} \mathbf{B}(x),
$$

where $\mathbf{B}(x)$ and $\mathbf{C}$ are defined in Eqs.(10) and (11). By using Eqs.(12) and (13) we have

$$
y^{\prime}(x)=\mathbf{C}^{T} \mathbf{B}^{\prime}(x)=\mathbf{C}^{T} \mathbf{D}^{(1)} \mathbf{B}(x),
$$

and

$$
y^{\prime \prime}(x)=\mathbf{C}^{T} \mathbf{B}^{\prime \prime}(x)=\mathbf{C}^{T}\left(\mathbf{D}^{(1)}\right)^{2} \mathbf{B}(x) .
$$

By substituting Eqs.(15), (16) and (17) in Eq. (1) we have

$$
\mathbf{C}^{T}\left(\mathbf{D}^{(1)}\right)^{2} \mathbf{B}(x)+\left(a+\frac{m}{x}\right) \mathbf{C}^{T} \mathbf{D}^{(1)} \mathbf{B}(x)=f\left(x, \mathbf{C}^{T} \mathbf{B}(x)\right) \cdot(18)
$$

Also by using Eqs.(2), (3), (15) and (16) we have

$$
\begin{aligned}
\alpha_{1} \mathbf{C}^{T} \mathbf{B}(0)+\beta_{1} \mathbf{C}^{T} \mathbf{D}^{(1)} \mathbf{B}(0) & =\gamma_{1}, \\
\alpha_{2} \mathbf{C}^{T} \mathbf{B}(1)+\beta_{2} \mathbf{C}^{T} \mathbf{D}^{(1)} \mathbf{B}(1) & =\gamma_{2} .
\end{aligned}
$$

Eqs.(19) and (20) give two linear equations. Since the total unknowns for vector $\mathbf{C}$ in Eq.(15) is $(m+1)$, we collocate Eq.(18) in $(m-1)$ points $x_{i}$ in the interval $[0,1]$ that are roots of shifted Legendre polynomial $P_{m-1}$, then we have,

$$
\mathbf{C}^{T}\left(\mathbf{D}^{(1)}\right)^{2} \mathbf{B}\left(x_{i}\right)+\left(a+\frac{m}{x_{i}}\right) \mathbf{C}^{T} \mathbf{D}^{(1)} \mathbf{B}(x)=f\left(x_{i}, \mathbf{C}^{T} \mathbf{B}\left(x_{i}\right)\right),
$$

for $i=1, \ldots, m-1$. Now the resulting Eqs. (19), (20) and (21) generate a system of $(m+1)$ nonlinear equations which can be solved using Newton's iterative method[24,25]. We used the Mathematica 7 software to solve this nonlinear system. 


\section{Illustrative Examples and Applied Models}

To show the efficiency of the proposed numerical method, we implement it on three nonlinear singular boundary problems that arise in real physiology applications. Our results are compared with result in Refs.[17-20]. The austerity of our method in implementation in analogy to other existed methods and its trusty answers is considerable.

\subsection{Example 1}

Consider the following oxygen diffusion problem

$$
y^{\prime \prime}(x)+\frac{2}{x} y^{\prime}(x)=\frac{0.76129 y}{y+0.03119},
$$

with the boundary conditions:

$$
y^{\prime}(0)=0, \quad 5 y(1)+y^{\prime}(1)=5 .
$$

Table 1 shows the numerical results for various number of meshes, and present method solutions are compared with results in Refs.[17] and[18].

Table 1. Approximate solutions for Example 1.

\begin{tabular}{|c|c|c|c|}
\hline$x$ & $\begin{array}{c}\text { Present method } \\
\text { with } m=15\end{array}$ & $\begin{array}{c}\text { Method in[17] } \\
\text { With } n=20\end{array}$ & $\begin{array}{c}\text { Method in[18] } \\
\text { with } n=60\end{array}$ \\
\hline 0.0 & 0.82848329035968 & 0.82848329481355 & 0.82848327295802 \\
\hline 0.1 & 0.82970609243380 & 0.82970609688790 & 0.82970607521884 \\
\hline 0.2 & 0.83337473359100 & 0.83337473804308 & 0.83337471691089 \\
\hline 0.3 & 0.83948991395370 & 0.83948991833986 & 0.83948989814383 \\
\hline 0.4 & 0.84805278499606 & 0.84805278876051 & 0.84805277036165 \\
\hline 0.5 & 0.85906492716923 & 0.85906492753032 & 0.85906491397434 \\
\hline 0.6 & 0.87252831995828 & 0.87252831569855 & 0.87252830841853 \\
\hline 0.7 & 0.88844530562319 & 0.88844529949702 & 0.88844529589927 \\
\hline 0.8 & 0.90681854806680 & 0.90681854179965 & 0.90681854026297 \\
\hline 0.9 & 0.92765098836558 & 0.92765098305256 & 0.92765098252660 \\
\hline 1.0 & 0.95094579849648 & 0.95094579480523 & 0.95094579461056 \\
\hline
\end{tabular}

Table 2. Numerical errors for Example 2.

\begin{tabular}{|c|c|c|c|}
\hline$x$ & $\begin{array}{c}\text { Present method } \\
\text { with } m=15\end{array}$ & $\begin{array}{c}\text { Present method } \\
\text { with } m=20\end{array}$ & $\begin{array}{c}\text { Approach II[17] } \\
\text { with } n=20\end{array}$ \\
\hline 0.0 & $3.88 \times 10^{-16}$ & $2.22 \times 10^{-16}$ & $2.00 \times 10^{-6}$ \\
\hline 0.1 & $3.88 \times 10^{-16}$ & $3.33 \times 10^{-16}$ & $1.99 \times 10^{-6}$ \\
\hline 0.2 & $3.33 \times 10^{-16}$ & $4.99 \times 10^{-16}$ & $1.97 \times 10^{-6}$ \\
\hline 0.3 & $3.88 \times 10^{-16}$ & $2.77 \times 10^{-16}$ & $1.94 \times 10^{-6}$ \\
\hline 0.4 & $2.22 \times 10^{-16}$ & $3.88 \times 10^{-16}$ & $1.83 \times 10^{-6}$ \\
\hline 0.5 & $6.10 \times 10^{-16}$ & $4.44 \times 10^{-16}$ & $1.78 \times 10^{-6}$ \\
\hline 0.6 & $8.32 \times 10^{-17}$ & $8.32 \times 10^{-17}$ & $1.67 \times 10^{-6}$ \\
\hline 0.7 & $4.44 \times 10^{-16}$ & $3.33 \times 10^{-16}$ & $1.34 \times 10^{-6}$ \\
\hline 0.8 & $8.32 \times 10^{-17}$ & $2.35 \times 10^{-16}$ & $9.20 \times 10^{-7}$ \\
\hline 0.9 & $1.94 \times 10^{-16}$ & $1.24 \times 10^{-16}$ & $4.57 \times 10^{-7}$ \\
\hline 1.0 & $1.96 \times 10^{-17}$ & $7.11 \times 10^{-18}$ & 0 \\
\hline
\end{tabular}

\subsection{Example 2}

Consider the following singular two point boundary value problem:

$$
\begin{aligned}
& y^{\prime \prime}(x)+\frac{1}{x} y^{\prime}(x)=-e^{y}, \\
& y^{\prime}(0)=0, \quad y(1)=0,
\end{aligned}
$$

with the exact solution

$$
y(x)=2 \ln \left(\frac{c+1}{c x^{2}+1}\right),
$$

where $c=3-2 \sqrt{2}$. Table 2 shows numerical errors of this example in analogy to errors for this example in[17].

\subsection{Example 3}

Consider this problem that is coincide by heat conduction model of the human head,

$$
y^{\prime \prime}(x)+\frac{2}{x} y^{\prime}(x)=-e^{-y},
$$

we consider the solution of this problem with conditions as follows:

$$
y^{\prime}(0)=0, \quad y(1)+y^{\prime}(1)=0 \text {. }
$$

Table 3 illustrates results for this example by proposed method alongside numerical solutions for this example that have been given in Refs[19-20].

Table 3. Approximate solutions for Example 3.

\begin{tabular}{|c|c|c|c|}
\hline$x$ & $\begin{array}{c}\text { Present method } \\
\text { with } m=15\end{array}$ & $\begin{array}{c}\text { Method in[19] } \\
\text { with forth-order }\end{array}$ & $\begin{array}{c}\text { Method } \\
\text { in[20] }\end{array}$ \\
\hline 0.0 & 0.3675168151 & 0.3675181074 & 0.3675169710 \\
\hline 0.1 & 0.3663623292 & 0.3663637561 & 0.3663623697 \\
\hline 0.2 & 0.3628940661 & 0.3628959378 & 0.3628941066 \\
\hline 0.3 & 0.3570975457 & 0.3570991429 & 0.3570975842 \\
\hline 0.4 & 0.3489484206 & 0.3489499903 & 0.3489484612 \\
\hline 0.5 & 0.3384121487 & 0.3384136581 & 0.3384121893 \\
\hline 0.6 & 0.3254435224 & 0.3254450019 & 0.3254435631 \\
\hline 0.7 & 0.3099860402 & 0.3099878567 & 0.3099860810 \\
\hline 0.8 & 0.2919711030 & 0.2919789654 & 0.2919711440 \\
\hline 0.9 & 0.2713170101 & 0.2713185637 & 0.2713170512 \\
\hline 1.0 & 0.2479277233 & 0.2479292837 & 0.2479277646 \\
\hline
\end{tabular}

Table 4. The maximum absolute errors in solution of Example 4 for $h=0.25, h=0.75$

\begin{tabular}{|c|c|c|c|c|}
\hline$m$ & $\begin{array}{c}\text { Case (i) } \\
h=0.25\end{array}$ & $\begin{array}{c}\text { Case (i) } \\
h=0.75\end{array}$ & $\begin{array}{c}\text { Case (ii) } \\
h=0.25\end{array}$ & $\begin{array}{c}\text { Case (ii) } \\
h=0.75\end{array}$ \\
\hline 15 & $1.39 \times 10^{-5}$ & $1.66 \times 10^{-5}$ & $3.67 \times 10^{-5}$ & $3.49 \times 10^{-5}$ \\
\hline 20 & $2.98 \times 10^{-8}$ & $2.06 \times 10^{-9}$ & $2.94 \times 10^{-8}$ & $2.97 \times 10^{-8}$ \\
\hline 25 & $8.88 \times 10^{-16}$ & $6.66 \times 10^{-16}$ & $2.22 \times 10^{-15}$ & $1.55 \times 10^{-15}$ \\
\hline 30 & $8.88 \times 10^{-16}$ & $8.88 \times 10^{-16}$ & $6.66 \times 10^{-16}$ & $8.88 \times 10^{-16}$ \\
\hline
\end{tabular}

Table 5. The maximum absolute errors in solution of Example 4 for $h=1, h=2$.

\begin{tabular}{|c|c|c|c|c|}
\hline$m$ & $\begin{array}{c}\text { Case (i) } \\
h=1\end{array}$ & $\begin{array}{c}\text { Case (i) } \\
h=2\end{array}$ & $\begin{array}{c}\text { Case (ii) } \\
h=1\end{array}$ & $\begin{array}{c}\text { Case (ii) } \\
h=2\end{array}$ \\
\hline 15 & $1.50 \times 10^{-5}$ & $7.78 \times 10^{-6}$ & $3.51 \times 10^{-5}$ & $3.70 \times 10^{-5}$ \\
\hline 20 & $9.06 \times 10^{-10}$ & $1.80 \times 10^{-10}$ & $2.99 \times 10^{-8}$ & $3.08 \times 10^{-8}$ \\
\hline 25 & $1.33 \times 10^{-15}$ & $2.39 \times 10^{-14}$ & $1.33 \times 10^{-15}$ & $8.88 \times 10^{-16}$ \\
\hline 30 & $8.88 \times 10^{-16}$ & $1.84 \times 10^{-14}$ & $6.66 \times 10^{-16}$ & $6.66 \times 10^{-16}$ \\
\hline
\end{tabular}

\subsection{Example 4}

Consider the following singular two point boundary value problem:

$$
y^{\prime \prime}(x)+\left(1+\frac{h}{x}\right) y^{\prime}(x)=\frac{5 x^{3}\left(5 x^{5} e^{y}-h-4-x\right)}{4+x^{5}},
$$

for the following two cases: 
(i) $y(0)=\ln \left(\frac{1}{4}\right), \quad y(1)+5 y^{\prime}(1)=\ln \left(\frac{1}{5}\right)-5$,

(ii) $y^{\prime}(0)=0, \quad y(1)+5 y^{\prime}(1)=\ln \left(\frac{1}{5}\right)-5$,

with the exact solution

$$
y(x)=\ln \left(\frac{1}{4+x^{5}}\right) .
$$

Maximum absolute errors for this problem have been displayed for $h<1$ in Table 4 and for $h \geq 1$ in Table 5, which show the accuracy of proposed method and these results in analogy to exhibited results for this example in[19-20] show advantage of this method.

\section{Conclusions}

This paper present a new approach, based on shifted Legendre polynomials for the numerical solution of a class of singular boundary value problems arising in biology and physiology problems. By use of shifted Legendre polynomials as basis and operational matrices of derivatives of these functions we convert such problems to an algebraic system. The implementation of current approach in analogy to existed methods is more convenient and the accuracy is high and we can execute this method in a computer speedy with minimum CPU time used. The numerical applied models that have been presented in the paper and the compared results support our claim.

\section{ACKNOWLEDGEMENTS}

The authors would like to thank Islamic Azad University of Karaj Branch for partially financially supporting this re-search and providing facilities and encouraging this work.

\section{REFERENCES}

[1] J.A. Adam, A simplified mathematical model of tumor growth, Math. Biosci., vol.81, pp.224-229, 1986

[2] J.A. Adam, A mathematical model of tumor growth II: effect of geometry and spatial non-uniformity on stability, Math. Biosci. vol.86, pp.183-211, 1987

[3] J.A. Adam, S.A. Maggelakis, Mathematical model of tumor growth IV: effect of necrotic core, Math. Biosci., vol. 97, pp. 121-136, 1989

[4] A.C. Burton, Rate of growth of solid tumor as a prob-lem of diffusion, Growth, vol.30, pp.157-176, 1966

[5] H.P. Greenspan, Models for the growth of solid tumor as a problem by diffusion, Stud. Appl. Math., vol.52, pp.317-340, 1972

[6] N.S. Asaithambi, J.B. Goodman, Point wise bounds for a class of singular diffusion problems in physiology, Appl. Math. Comput., vol. 30 , pp.215-222, 1989

[7] H.S. Lin, Oxygen diffusion in a spherical cell with non-linear oxygen uptake kinetics, J. Theor. Biol., vol. 60 , pp.449-457, 1976

[8] D.L.S. McElwain, A re-examination of oxygen diffusion in a spherical cell with MichaelisMenten oxygen uptake kinetics, J. Theor. Biol., vol. 71, pp.255-263, 1978

[9] N. Rashevsky, Mathematical Biophysics, vol. 1, Dover, New York, 1960

[10] U. Flesch, The distribution of heat sources in the hu-man head: a theoretical consideration, J. Theor. Biol., vol. 54, pp. 285-287, 1975

[11] J.B. Garner, R. Shivaji, Diffusion problems with mixed nonlinear boundary condition, J. Math. Anal. Appl., vol. 148, pp. 422-430, 1990

[12] B.F. Gray, The distribution of heat sources in the hu-man head: a theoretical consideration, J. Theor. Biol., vol. 82 pp. 473-476, 1980

[13] R.C. Duggan, A.M. Goodman, Point wise bounds for nonlinear heat conduction model for the human head, Bull. Math. Biol., vol. 48 (2), pp. 229-236, 1989

[14] R.K. Pandy, On a class of weakly regular singular two point boundary value problems II, J, Differential Equa-tions, vol. 127, pp. 110-123, 1996

[15] M.M. Chawla, P.N. Shivkumar, On the existence of so-lution of a class of singular two--point nonlinear boun-dary value problems, J. Comput. Appl. Math., vol. 19, pp. 379-388, 1987

[16] R.D. Russell, L.F. Shampine, Numerical methods for singular boundary value problems, SIAM J. Numer. Anal., vol. 12, pp. 13-36, 1975

[17] S.A. Khuri, A. Sayfy, A novel approach for the solution of a class of singular boundary value problems arising in physiology, J. Math. Comput. Model., vol. 52, pp. 626-636, 2010

[18] Hikmet Caglar, Nazan Caglar, Mehmet Ozer, B-spline solution of non-linear singular boundary value problems arising in physiology, Chaos Solitons Fractals, vol. 39, pp. 1232-1237, 2009

[19] J. Rashidinia, R. Mohammadi, R. Jalilian, The numerical solution of non-linear singular boundary value problems arising in physiology, J. Appl. Math. Comput., vol. 185, pp. 360-367, 2007

[20] R.K. Pandey, Arvind K. Singh, On the convergence of a finite difference method for a class of singular boundary value problems arising in physiology, J. Comput. Appl. Math., vol. 166, pp. 553-564, 2004

[21] A. Saadatmandi, M. Dehghan, A new operational ma-trix for solving fractional-order differential equations, J. Comput. Math. Appl., vol. 59, pp. 1326-1336, 2010

[22] K. Maleknejad, B. Basirat, E. Hashemizadeh, Hybrid Le-gendre polynomials and Block-Pulse functions approach for nonlinear Volterra-Fredholm integro-differential eq-uations, Comput. Math. Appl.,vol. 61, pp. 2821-2828, 2011

[23] K. Maleknejad, E. Hashemizadeh, A numerical approach for Hammerstein integral equations of mixed type using operational matrices of hybrid functions, Scientific Bulle-tin, Series A: Applied Mathematics and Physics, vol .73)3(, pp. 95-104, 2011 
[24] K. Maleknejad, S. Sohrabi, H. Derili, A new computa-tional method for solution of non-linear Volter-ra-Fredholm integro-differential equations, Int. J. Appl. Math., vol. 87(2), pp. 327-338, 2010

[25] K. Maleknejad, E. Hashemizadeh, B. Basirat, Computational method based on Bernestein operational matrices for nonlinear Volterra-Fredholm-Hammerstein integral equations, Commun. Nonlinear. Sci. Numer. Simulat., vol. 17, pp.52-61, 2012 